\title{
Numerical Analysis of Wing Vortex Aerodynamic Characteristics
}

\author{
Hangxing Zhou ${ }^{1 *}$ and Changjiang Sun ${ }^{1}$ \\ ${ }^{1}$ Zhuhai College of Beijing Institute of Technology \\ *yuyangtao312@163.com
}

Keywords: Wingtip vortices; Angle of attack; Aerodynamic characteristics; N-S equation

\begin{abstract}
Influence of aircraft wingtip vortices will make the lift coefficient of formation flying wingman decreases, the drag coefficient increases, the lift drag ratio decreased sharply, attack decreased the stall angle of attack. To correctly reflect the basic information around the airfoil flow field of aircraft, and reveal the influence degree of these performance parameters on the control performance of aircraft, a numerical method is adopted in this paper to analyze the aerodynamic characteristics and maneuverability of aircraft. Based on the in-depth study of the N-S equation of low speed viscous flow in Western Europe and the United States, a method based on the improved simple numerical model for solving the N-S equation is proposed. The method takes into account the viscous effect of the flow field during the flight, and obtains the numerical simulation results of the flow field.
\end{abstract}

\section{Origin of Wing Tip Vortex}

Wing tip vortex Is a kind of pressure phenomenon. Because of the pressure difference on the surface of the wing, the tip air flow will turn upside down under pressure and form a spiral flow with cusp as the center, backward and high speed, and downward downward. The two wings of an aircraft are formed by the rotation direction of spiral vortices in the opposite direction. The formation flight passes through the front vortex area and will be affected by the air flow. Vortex intensity is mainly affected by the wing structure, the quality of the aircraft, flight speed and flight angle will also affect the strength of the aircraft, the aircraft is the main angle of attack. The tip vortices spread out and spread downward. After the formation of the vortex 120 to 150 meters / minute rate decline in aircraft wingtip vortex generation to 250 meters to the level of the effect disappears. When the side wind is flying, the vortex moves down in the direction of the side wind. The improper disposal of the pilot will change the flight status of the aircraft, even in the seriousness of the roll.

\section{Wingtip flow Field Aerodynamic Characteristics Analysis}

Reasonable and accurate prediction of wingtip spoiler affected zone is to give the accurate control of response judgment premise. Domestic and foreign scholars from the 90's of the last century, began to carry out a lot of research on the flow field prediction method of the wing spoiler, draw many conclusions have guiding significance. This paper on the basis of these results, combined with the inherent characteristics of a certain type of airplane to the process of turbulence diffusion hypothesis is a quasi stationary process, namely the disturbed flow area is a layer of dissipation, the time step is the Yi long walks.

Atmospheric turbulence field generated in accordance with the time domain correlation characteristics of turbulent flow model. For inspection and the effectiveness of the three-dimensional turbulent flow field, this paper examined the turbulent flow field of the one dimensional correlation and two-dimensional cross correlation. Three-dimensional turbulent flow field for isotropic: in the process of flight simulation, to simulate continuous plane through the turbulent flow field, turbulent flow field of the space extension problems inevitably. To this, some scholars put forward a kind of simple method, the plane in the position of the track coordinates, the mathematical expressions as the following: 
$\mathrm{x}_{\mathrm{E}}^{\prime}=\mathrm{x}_{\mathrm{E}} \operatorname{modr}_{1}$
$\mathrm{y}_{\mathrm{E}}^{\prime}=\mathrm{y}_{\mathrm{E}} \operatorname{modr}_{2}$
$\mathrm{z}_{\mathrm{E}}^{\prime}=\mathrm{z}_{\mathrm{E}} \operatorname{modr}_{3}$

The mod to take more than symbols. In this way can make the plane in any space motion, the turbulent flow field uninterrupted, realize "head-tail". There are some problems in the actual inspection. Therefore, this article take the following steps to improve.

Coordinates. For the need of modeling, the dynamic model USES the following basic assumptions:

To simplify the earth as the plane of the earth, without considering the earth curvature and rotation, the assumption for the inertial system earth. For the flight only at high subsonic speed range of large transport aircraft, precision is enough.

Ignore the gyro effect of rotating machinery.

In dynamic model is set up, the conventional layout of Oxz plane symmetry plane equation is established. Several typical coordinate system established by the before establishing equation: Coordinate system transformation relationship between each, as shown in Fig.1.

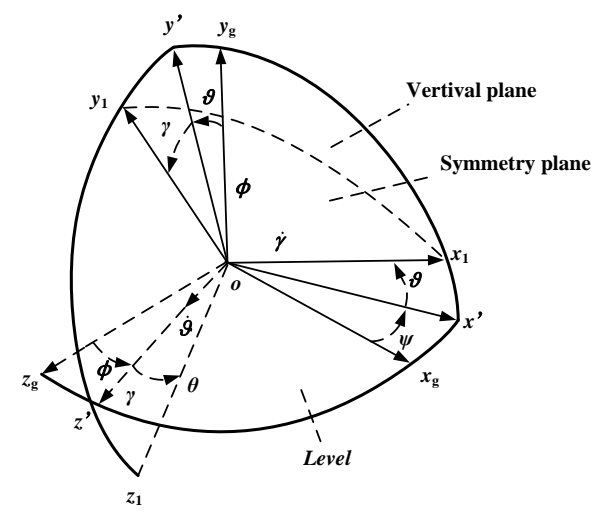

Figure 1. Coordinate system transformation relationship diagram

Flow Field of Aircraft Wing. For a certain type of aircraft aerodynamic characteristics and control effect of the starting point of the study is to describe the flow field, numerical flow around the wing. In the process of building a numerical model, the viscous effect can be considered, and the surface element method of classical gas dynamics can not meet the requirement of accurate calculation of flow field. In this study, the N-S equation method is used to construct the numerical model of the flow field, and the governing equations can be written in the Cartesian coordinate system:

$$
\frac{\partial \rho \phi}{\partial \mathrm{t}}+\nabla \cdot(\rho \mathrm{v} \phi-\Gamma \operatorname{grad} \phi)=\mathrm{q}
$$

Continuity equation:

$\phi=1, \quad \Gamma=\mu, \mathrm{q}=0$

$\mathrm{X}$ direction momentum equation:

$\phi=\mathrm{u}, \quad \Gamma=\mu, \mathrm{q}=-\frac{\partial \mathrm{P}}{\partial \mathrm{x}}+\nabla\left(\mu \frac{\partial \mathrm{v}}{\partial \mathrm{x}}\right)+\rho \mathrm{f}_{\mathrm{x}}$

Y direction momentum equation: 


$$
\phi=\mathrm{v}, \quad \Gamma=\mu, \mathrm{q}=-\frac{\partial \mathrm{P}}{\partial \mathrm{y}}+\nabla\left(\mu \frac{\partial \mathrm{v}}{\partial \mathrm{y}}\right)+\rho \mathrm{f}_{\mathrm{y}}
$$

$\mathrm{Z}$ direction momentum equation:

$$
\phi=\mathrm{w}, \quad \Gamma=\mu, \quad \mathrm{q}=-\frac{\partial \mathrm{P}}{\partial \mathrm{z}}+\nabla\left(\mu \frac{\partial \mathrm{v}}{\partial \mathrm{z}}\right)+\rho \mathrm{f}_{\mathrm{z}}
$$

Because during actual flight of the aircraft, the wing disturbance flow is a turbulent environment, so numerical modeling process need to take into account the turbulence, this study substituted into the standard k-e turbulence model to simulate the effects of actual flight environment turbulence, the turbulent kinetic energy equation:

$$
\phi=\mathrm{k}, \quad \Gamma=\mu_{1}+\frac{\mu_{\mathrm{t}}}{\delta_{\mathrm{k}}}, \quad \mathrm{q}=\mathrm{G}_{\mathrm{k}}-\rho \varepsilon+\mathrm{G}_{\mathrm{b}}
$$

Turbulent energy dissipation rate equation:

$$
\phi=\varepsilon, \quad \Gamma=\mu_{1}+\frac{\mu_{\mathrm{t}}}{\delta_{\varepsilon}}, \quad \mathrm{q}=\frac{\varepsilon}{\mathrm{k}}\left(\mathrm{C}_{1} \mathrm{C}_{\mathrm{k}}-\mathrm{C}_{2} \rho \varepsilon\right)
$$

The convection term and diffusion term are obtained by using explicit treatment, and the source term is put into the source term. In order to make the source term discrete as far as possible to reflect the actual flow field, the step size is not easy to be too large, it is the amount of variation as far as possible to approximate the initial value of the source term. In the numerical treatment of link, according to certain rules are given a discrete equation form, the equation of the main diagonal dominance has been strengthened, the flow field numerical simulation equations iteratively solving the stability of favorable, so it is necessary to expression of source term linearization, according to momentum equation of the flow field in the model can be $u$ equation with a source term is expressed as:

$$
\mathrm{a}_{\mathrm{u}}=-\int_{\mathrm{v}} \frac{\partial \mathrm{P}}{\partial x} d V+\oint_{A}\left(\mu \frac{\partial v}{\partial x}\right) \cdot d A
$$

In summary, the flow field around the airfoil can be obtained by solving the discrete equations by solving the algebraic equations. The flow field information of a certain type of aircraft wing is shown in Fig. 2.

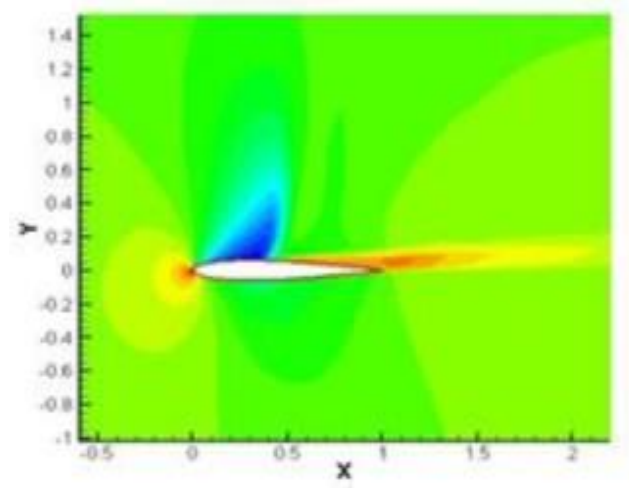

Figure 2. The flow field information of a certain type of aircraft wing

\section{Response of Tip Vortex Model of Aircraft}

A certain type of aircraft wingtip vortex response model is an important basis for determining the formation flight range. In the present experiment space prone to the informer of flight is not conducive 
to security conditions, the aircraft design and flight eddy current environment platform of virtual prototype to verify, continue to refine the formation flight control, can also under different meteorological conditions hold special range provide the dynamic analysis method.

In high precision, it is necessary to use closed-loop control model. At that time it is needed to improve the aircraft wingtip vortex flow field open loop simulation system structure. As shown in Fig.3, in the analysis of vortex flow field of machine gas dynamic effect in the process of, after the introduction of machine bullied influence of attack angle feedback data information to form a closed loop system analysis. After introducing the angle feedback information, the condition of the termination calculation will feedback the information which need be judged. In order to achieve this function, a separate judgment sub module is set in the structure of the closed loop system StopSim. The formation machine angle of attack and the mean value of the response is used as a feedback signal, substitution system model loop calculation, when the response speed, gas velocity and the absolute value of the difference less than stop judgment value, end of operation, system output speed response value.

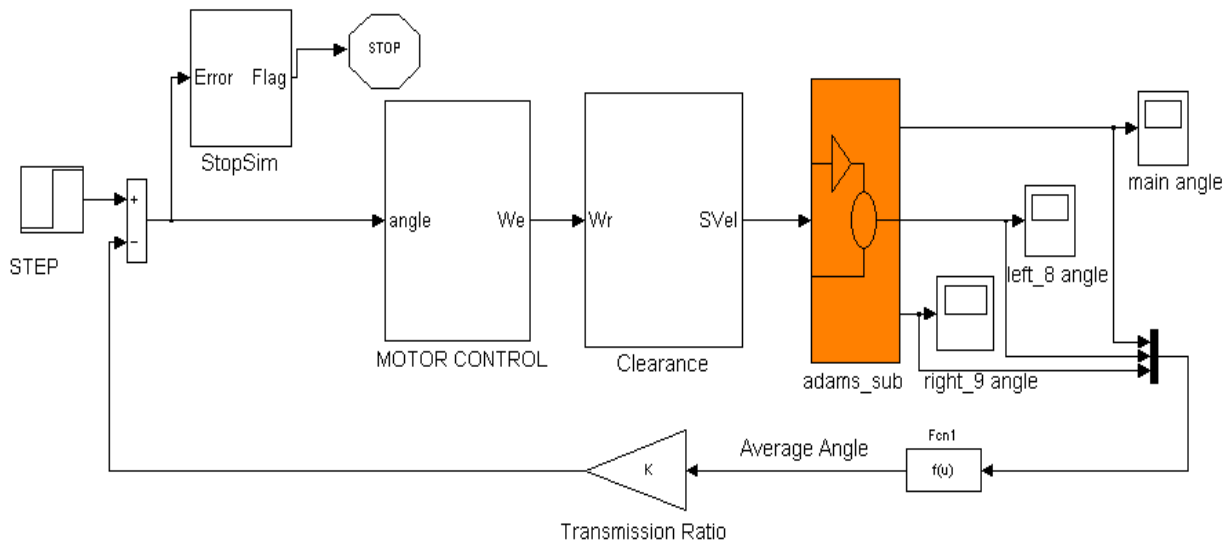

Figure 3. MATALAB closed-loop control

Accompanied by wingtip vortices, the machine in motion process has a slight jitter phenomenon, which is due to the eddy current flow direction on the machine under the pressure function, then in the front position of the vortex, the roll after the captain; in addition, because of the vortex flow trajectories in the horizontal plane was s shape, so on machine milling effect also showed to center deviation phenomenon. When the distance reaches a certain degree, the wingtip vortex aerodynamic effect will be very attenuation. The wingtip vortex aerodynamic effect is as Fig.4.

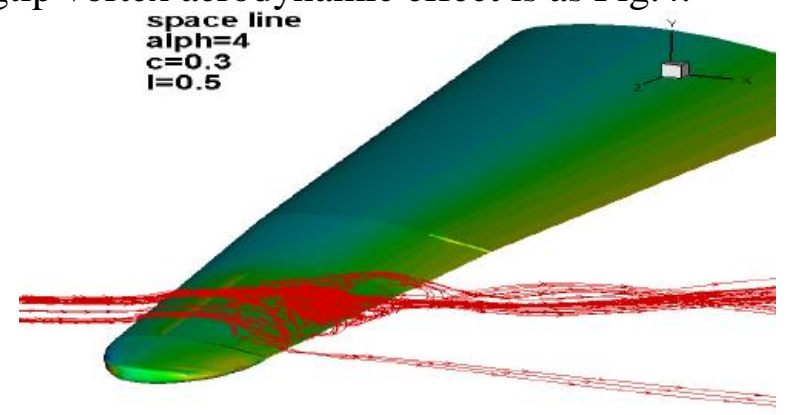

Figure 4. Three dimensional simulation of tip vortex

\section{Conclusion}

This research mainly carried on the following work:

On the basis of studying the existing theory of computational fluid dynamics, the N-S equations are 
solved to solve the flow field around the aircraft;

Using ANSYS finite element analysis software to analyze the aerodynamic characteristics of aircraft;

\section{References}

[1] Yi Xian, Iced airfoil numerical simulation and iced airfoil of gas dynamic characteristics analysis [D]. Beijing: China Aerodynamics Research and Development Center (doctoral dissertation, 2002:35-42.

[2] Li Shumin, low speed viscous flow calculation method and Application Research [D]. China Aerodynamics Research and development center doctoral dissertation, 2004:16-22.

[3] Guo Bin, Xiong ray, Chen Xiaobo, et al. Research and implementation of universal adapter MATLAB and [J]. HLA/RTI Journal of system simulation, 2004, 16 (6): 1275-1279.

[4] Yi Xian, Zhu Guolin, Liu Zhitao, Chen Hong. Ice accretion on numerical prediction and icing test similar criterion $[\mathrm{J}]$. The second session of the China Aviation society youth science and Technology Forum corpus. Aviation Industry Press.2006,5:112-120. 